\title{
PAPERS
}

PUBLISHED IN THE

\section{PROCEEDINGS OF THE LONDON MATHEMATICAL SOCIETY}

\author{
ON THE ARITHMETICAL NATURE OF THE COEFFICIENTS IN \\ A GROUP OF LINEAR SUBSTITUTIONS OF FINITE ORDER \\ (Second Paper)
}

By W. Burnside.

[Received October 12th, 1905.-Read November 9th, 1905.]

IN a former paper dealing with this question (Proc. London Math. Soc., Series 2, Vol. 3, pp. 239-252) the subject was approached from the point of view of the reduction of the group when represented in the form of a group of permutations. One of the results arrived at was to determine a condition subject to which it is possible to exhibit an irreducible group of finite order in a form in which the coefficients are rational functions of the characteristics; but the question as to whether this condition was generally satisfied was not dealt with.

In the present paper the problem is approached from a different and, so to say, a more self-contained point of view.

An irreducible group of linear substitutions of finite order is supposed to be given in any one of its possible forms, without any assumption at all as to the nature of the coefficients. With regard to such a group the question arises as to whether it is possible to choose new variables, so that, when expressed in terms of them, the coefficients of the substitutions belong to an assigned domain of rationality. If the domain of rationality does not contain the characteristics of the group, it is certainly impossible to do this. The simplest domain of rationality to which the coefticients can belong is that defined by the characteris tics.

8BR. 2. VOL. 4 . No. 912 . 
It is shown here, by direct considerations, in which the theory of the reduction of the group, regarded as a permutation-group, is not introduced, that in general it is possible to exhibit the group so that the coefficients belong to the domain of rationality defined by the characteristics. There are exceptions to this general rule, and the whole of the exceptions are not here determined; but it is, I think, made clear that the cases which do not come under the rule are actually of the nature of exceptions.

1. I consider a discontinuous irreducible group of linear substitutions in $m$ variables. The group itself is denoted by $\Gamma$, and any substitution of the group

$$
x_{i}^{\prime}=\sum_{j=1}^{j=m} s_{i j} x_{j} \quad(i=1,2, \ldots, m)
$$

is denoted by $S$. The sum of the coefficients in the leading diagonal of $S$, or its characteristic, is denoted by $\chi_{s}$. Such a group necessarily contains a set of $m^{2}$ substitutions $d^{(k)}\left(k=1,2, \ldots, m^{2}\right)$ which are linearly independent* in the sense that the determinant

is not zero.

$$
\begin{array}{lllr}
a_{11}^{(1)} & a_{12}^{(1)} & \ldots & a_{m m}^{(1)} \\
a_{11}^{(2)} & a_{12}^{(2)} & \ldots & a_{m m}^{(2)} \\
\ldots & \ldots & \ldots & \ldots \\
a_{11}^{\left(m^{2}\right)} & a_{12}^{\left(m^{2}\right)} & \ldots & a_{m m}^{\left(m^{2}\right)}
\end{array}
$$

This being the case, the equations

$$
s_{i j}=\sum_{k=1}^{k=m^{2}} a_{i j}^{(k)} S_{k} \quad(i, j=1,2, \ldots, m)
$$

are linearly independent, and determine the $m^{2}$ quantities $S_{k}$. The system of $m^{2}$ equations (i) may be replaced by

$$
\sum_{i, j} s_{i j} a_{j i}^{(i)}=\sum_{k=1}^{k=m^{2}} S_{k} \sum_{i j} a_{i j}^{(i)} a_{j i}^{(l)} \quad\left(l=1,2, \ldots, m^{2}\right) .
$$

But $\sum_{i j} s_{i j} a_{j i}^{(l)}$ is the characteristic of $S A^{(l)}$. Hence the $m^{2}$ quantities $S_{k}$ are determined by

$$
\chi_{S A}{ }^{n j}=\sum_{1}^{m^{2}} S_{k} \chi_{A^{(k)} A^{(l)}},
$$

and they are therefore rational functions of the characteristics of the group. 
The $m^{2}$ equations (i) are equivalent to the single equation

$$
S=\sum_{1}^{m^{2}} A^{(k)} S_{k}
$$

expressing that the substitution (or matrix) $S$ is a linear function of the substitutions (or matrices) $A^{(k)}$; and the coefficients which enter in this expression for $S$ are rational functions of the characteristics of the group.

If $S, T, U$ are three substitutions of the group, such that

$$
S T=U,
$$

then

$$
\Sigma A^{(k)} U_{k}=\Sigma A^{(k)} S_{k} \Sigma A^{(l)} T_{l} .
$$

But, by (ii), $A^{(k)} A^{(l)}$ can be expressed linearly in terms of the $m^{2}$ independent substitutions. Let

$$
A^{(k)} A^{(l)}=\Sigma A^{(p)} \alpha_{k l p}
$$

be the equation so expressing it, the constants $\alpha_{k l p}$ being rational functions of the characteristics. Then (iii) may be written

$$
\sum_{p} A^{(p)} U_{p}=\sum_{k, l, p} a_{k l p} S_{k} T_{l} A^{(p)} .
$$

The $m^{2}$ substitutions $A^{(p)}$ being linearly independent, this relation among the substitutions (or matrices) is equivalent to the system of $\mathrm{m}^{2}$ equations

$$
U_{p}=\sum_{k, l} \alpha_{k l p} S_{k} T_{l} \quad\left(p=1,2, \ldots, m^{2}\right) .
$$

Now the relation

$$
S T=U
$$

is also equivalent to the system of $m^{2}$ equations

$$
u_{i j}=\sum_{k} t_{i k} s_{k j} \quad(i, j=1,2, \ldots, m)
$$

between the coefficients of the three substitutions $S, T$, and $U$. Hence equations (v) is the form which equations (vi) take when the $s$ 's, $t$ 's, and $u$ 's are cogrediently replaced by new symbols, $S$ 's, T's, and $U$ 's, defined by the equations (i).

But, if the $s$ 's are regarded as the original and the $u$ 's as the transformed variables, while for the $t$ 's are taken in turn the coefficients of each substitution of $\Gamma$, then equations (vi) define a group of linear substitutions on $m^{2}$ variables which is simply isomorphic with $\Gamma$. Moreover in this group each set of $m$ symbols with the same second suffix undergoes for each substitution of the group exactly the same transformation as the original $x$ 's; and there are $m$ such sets. The group may then be denoted by $m \Gamma$, implying that $m$ distinct sets of variables each undergo cogrediently the substitutions of the original group $\Gamma$. 
If then, in (v), the $S$ 's are regarded as the original and the $U$ 's as the transformed variables, while the $T$ 's take in turn the values corresponding to each of the substitutions of $\Gamma$, the resulting system of equations is a form into which the group $m \Gamma$ can be thrown by a suitable choice of new variables. But the 'T's which correspond to the substitutions of $\Gamma$ and the coefficients $\alpha_{k l p}$ are rational functions of the characteristics of $\Gamma$. Hence $m \Gamma$ can be exhibited in a form in which all the coefficients are rational functions of the characteristics. Further, if in equations (vi) the $t$ 's are regarded as the original and the $u$ 's as the transformed variables, while for the $s$ 's the coefficients of each substitution of $\Gamma$ are taken in turn, then the equations give another form of $m \Gamma$, every one of whose substitutions is permutable with every substitution of the previous form. Hence the same is also true of equations (v) when the $T$ 's are taken as the original and the $U$ 's as the transformed variables. The results of this paragraph may then be summed up as follows :-

Theorem.-If $\Gamma$ is a discontinuous irreducible group of linear substitutions in $m$ variables, and if: $m \Gamma$ represents the group in $m^{2}$ variables that arises by carrying out the substitutions of $\Gamma$ cogrediently on $m$ sets of $m$ variables each, then a group equivalent to $m \Gamma$ can be set up in two distinct forms on a set of $m^{2}$ variables, so that (i) the coefficients in each form are rational functions of the characteristics of $\Gamma$, and (ii) every substitution of the one form is permutable with every substitution of the other.

2. If $\Gamma$ is a group of finite order, it will in general contain sub-groups for which one or more linear functions of the variables are invariants. In a paper recently published in the Messenger of Mathematics (Vol. xxxv., pp. 5]-55) I have determined the nature of those exceptional cases in which the identical substitution of $\Gamma$ is the only one for which there are linear invariants. They are very limited in number, and may be arranged in three classes, of which-

(i) the quaternion group in two variables;

(ii) the group generated by

$$
\begin{array}{rll}
x^{\prime}=\omega x, & y^{\prime}=\omega^{2} y, & z^{\prime}=\omega^{4} z, \\
x^{\prime}=y, & y^{\prime}=z, & z^{\prime}=\alpha x, \\
\omega^{7}=1, & a^{3}=1 ;
\end{array}
$$

(iii) the group of order 120 in two variables which is multiply isomorphic with the icosahedral group,

are typical. 
Apart from these very special exceptions every group of linear substitutions of finite order has some sub-group, other then that consisting of the identical substitution only, for which one or more linear functions of the variables are invariant.

3. Let $\Gamma$ now denote any given irreducible group of finite order on $m$ variables. Further, let $G$ and $G^{\prime}$ denote the two forms in which $m \Gamma$ can be set up on $m^{2}$ variables

$$
x_{1}, x_{2}, \ldots, x_{m},
$$

so that (i) the coefficients of the substitutions of $G$ and $G^{\prime}$ are rational functions of the characteristics of $\Gamma$, and (ii) every substitution of $G$ is permutable with every substitution of $G^{\prime}$. Denote by $\xi_{1}$ a linear function

$$
\sum A_{i} x_{i}
$$

of the $m^{2}$ variables with arbitrary coefficients ; and by

$$
\xi_{1}, \xi_{2}, \ldots, \bar{\xi}_{n},
$$

the functions into which $\xi_{1}$ is transformed by the $n$ substitutions of a sub-group $H^{\prime}$, of order $n$, of $G^{\prime}$. Then

$$
\sum_{1}^{n} \xi_{t}
$$

is the most general linear function of the variables which is invariant for $H^{\prime}$. Unless $\Gamma$ belongs to one of the exceptional cases above mentioned, $G^{\prime}$ must have some sub-group $H^{\prime}$ for which $\sum_{1}^{n} \xi_{t}$ is not identically zero. If in $\Gamma$ the sub-group $H^{\prime}$ has $i$ independent linear invariants, then in $m \Gamma$ there are $m i$ linearly independent linear functions invariant for $H^{\prime}$. Hence of the coefficients of $A_{1}, A_{2}, \ldots, A_{m^{2}}$ in $\sum_{1}^{n} \xi_{t}$, just $m i$ are linearly independent. Now the coefficient of $A_{s}$ in $\sum_{1}^{n} \xi_{l}$ is a linear function of the $x$ 's with coefficients which are rational in the characteristics of $\Gamma$. Hence $m i$ linear functions of the $x$ 's with coefficients rational in the characteristics of $\Gamma$ may be formed, each of which is invariant for $H^{\prime}$; and no other linear function of the $x$ 's, which is linearly independent of these, is invariant for $H^{\prime}$.

Now every substitution of $G$ is permutable with every substitution of $H^{\prime}$. Hence the substitutions of $G$ must transform among themselves these $m i$ linear functions of the $x$ 's; and the coefficients in the group of linear substitutions which so arises are rational functions of 
the characteristics. This is equivalent to the statement that, unless $\Gamma$ is one of the exceptions above mentioned, it is always possible to find a number $i(<m)$ such that $i \Gamma$ can be exhibited in a form in which the coefficients are rational functions of the characteristics.

Denote by

$$
y_{1}, y_{2}, \ldots, y_{m i},
$$

the $m i$ above linear fanctions. Any substitution $S^{\prime}$ of $G^{\prime}$ will change these into $m i$ linearly independent functions

$$
z_{1}, z_{2}, \ldots, z_{m i} \text {. }
$$

These, and only these, are invariants for the sub-group $S^{\prime-1} H^{\prime} S^{\prime}$; and therefore these are transformed among themselves by every substitution of $G$. Moreover, since

$$
S^{\prime-1} S S^{\prime}=S,
$$

if $S$ is any substitution of $G$, the $z$ 's and the $y$ 's undergo, for each substitution of $G$, the same transformation. It may be the case that for certain substitutions $S^{\prime}$ of $G^{\prime}$, the $y^{\prime}$ 's are linear functions of the $z$ 's. This cannot be the case for every substitution of $G^{\prime}$, for then both $G$ and $G^{\prime}$ would transform the $y$ 's among themselves.

It may, however, happen that when $S^{\prime}$ is suitably chosen. some, but not all, linear functions of the $y$ 's can be expressed linearly in terms of the $z$ 's. If this is so, it is possible to determine coefficients $A_{s}$ and $B_{t}$ so that in

$$
\Sigma A_{s} y_{s}+\Sigma B_{t} z_{t}
$$

the coefficient of each $x$ is identically zero. The equations expressing this are linear in the $A$ 's and $B$ 's with coefficients rational in the characteristics. Hence the linear functions, if any, of the $y$ 's which can be expressed in terms of the $z$ 's have coefficients which are rational in the characteristics. But, since the $z$ 's are transformed among themselves by $G$, those linear functions of the $y$ 's which can be expressed linearly in terms of the $z$ 's are transformed among themselves by $G$. If there are $j$ of them, the $j$ functions will be transformed among themselves by $G$, and the coefficients in the substitutions are still rational in the characteristics. Further, since $\Gamma$ is irreducible, the number of symbols transformed among themselves by any component of $G$ must be a multiple of $m$.

Hence, if the $y^{\prime} \mathrm{s}$ and $z^{\prime} \mathrm{s}$ are not linearly independent, it is possible to form $m i^{\prime}\left(i^{\prime}<i\right)$ linear functions of the $x^{\prime}$ s, with coefficients which are rational in the characteristics, that are transformed among themselves by $G$, and the coefficients of the resulting substitutions are rational in the characteristics. 
This set of $m i^{\prime}$ functions may be represented by

$$
y_{1}, y_{2}, \ldots, y_{m i},
$$

and may be dealt with as the original set of $m i$ were.

In this way we finally arrive at a set of $m a$ linear functions of the $x$ 's ( $a$ being as small as possible) with coefficients rational in the characteristics, which are transformed among themselves by $G$, the coefficients of the substitutions satisfying the same condition.

Represent them by $y_{1}^{(1)}, y_{2}^{(1)}, \ldots, y_{m a}^{(1)}$.

A substitution $S^{\prime}$ of $G^{\prime}$ changes these into

$$
y_{1}^{(2)}, y_{2}^{(2)}, \ldots, y_{m a}^{(2)},
$$

and it is certainly possible to choose $S^{\prime}$ so that these are linearly independent of the preceding ones. If $m>2 a$, it must again be possible to take $S^{\prime \prime}$ in $G^{\prime}$ so that

$$
y_{1}^{(3)}, y_{2}^{(3)}, \ldots, y_{m a}^{(3)},
$$

into which the functions of the first line are changed by $S^{\prime \prime}$, are linearly independent of those in the first two lines. This process may be continued till a set of $m^{2}$ functions linearly equivalent to the original $x$ 's have been formed. Hence $a$ must be a factor of $m$. Moreover, if $m=a b$, the functions in each of the $b$ sets

$$
y_{1}^{(t)}, y_{2}^{(t)}, \ldots, y_{m a}^{(t)}, \quad(t=1,2, \ldots, b)
$$

undergo for each substitution of $G$ the same transformation, the coefficients being rational functions of the characteristics.

Consider now any sub-group of $\Gamma$ which has $k$ independent linear invariants. In $G^{\prime}$ the sub-group has $m k$ linear invariants; which may be denoted by

$$
z_{1}, z_{2}, \ldots, z_{m k}
$$

and by the process above used these may be expressed linearly in terms of the $m^{2} y$ 's, with coefficients which are rational in the characteristics. The number denoted by $a$ being, by supposition, as small as possible, $k$ is not less than $a$. If $k$ is greater than $a$, and if in the expression of the $z^{\prime}$ 's the $y^{(1)}$ 's occur, the $m a y^{(1)}$ 's may be eliminated from the equations which express the $z$ 's in terms of the $y$ 's. In the elimination other sets of $y$ 's besides the $y^{(1) ' s}$ may disappear; but there must remain $m(k-a)$ equations, giving $m(k-a)$ independent linear functions of the $z$ 's (with coefficients which are rational in the characteristics) in terms of certain remaining sets of $y$ 's. Since each set of 
$y$ 's are transformed among themselves by $G$, these $m(k-a)$ functions of the $z$ 's are transformed among themselves by $G$, and the coefficients in the substitution are rational in the characteristics. If $k-a$ is greater than $a$, this process may be continued. Hence, unless $k$ is a multiple of $a$, it will be possible to obtain a set of $m a^{\prime}\left(a^{\prime}<a\right)$ functions which are transformed among themselves by $G$ with coefficients rational in the characteristics; and this is contrary to the supposition made. This result is equivalent to the following:-

Theorem.-An irreducible group of linear substitutions of finite order can certainly be exhibited in a form in which the coefficients in the substitutions are rational functions of the characteristics, unless there is an integer $a(>1)$ such that the number of linear invariants for every subgroup is either zero or a multiple of $a$.

Corollary.-An irreducible group of linear substitutions in an odd prime number $p$ of variables can be exhibited in a form in which the coefficients are rational functions of the characteristics, except in the case of the class of soluble groups $\{S, P\}$, defined by

$$
\begin{array}{llll}
S \sim x_{1}^{\prime}=\beta x_{1}, & x_{2}^{\prime}=\beta^{s} x_{2}, & \ldots, & x_{p}^{\prime}=\beta^{s^{p-1}} x_{p} ; \\
T \sim x_{1}^{\prime}=x_{2}, & x_{2}^{\prime}=x_{3}, & \ldots, & x_{p}^{\prime}=\alpha x_{1},
\end{array}
$$

where $a$ is a $p^{a}$-th root of unity, $\beta$ an $m$-th ( $m$ prime to $p$ ) root of unity, and

$$
s^{p} \equiv 1(\bmod m) .
$$

In fact, when the number of variables is prime $a$ must be unity; and therefore the group can certainly be exhibited in the desired form, unless the identical substitution is the only one for which there are linear invariants. Such groups occur only among the second of the three classes mentioned above; and I have shown in a paper in the Messenger of Mathematics, ${ }^{*}$ immediately preceding the one quoted above, that their form is that just stated.

4. I wish to emphasize the point that the cases in which an irreducible group of linear substitutions of finite order cannot be exhibited in a form in which the coefficients are rational functions of the characteristics are of the nature of exceptions. 
The general rule, subject to certain definite classes of exceptions, is that such a mode of exhibiting the group is possible.

The exceptions corresponding to the case in which $a$ is zero I have actually determined. The familiar case of the quaternion group in two variables for which all the characteristics are rational, while any possible form of the group involves $\sqrt{ }(-1)$ in the coefficients, suggested the possible occurrence of other similar exceptions and indeed led to this investigation. Whether there are also exceptions corresponding to values of $a$ other than zero I cannot at present say. I have, however, examined a large number of groups for which the characteristics have been calculated, and in no case in which I have determined the corresponding multipliers have I come across an exception corresponding to a value of $a$ other than zero. 